\title{
Praxisbezüge und Praktika als Beitrag zur Erhöhung der Berufsbefähigung im Studium: Beispiele - Befunde - Perspektiven
}

\author{
Wilfried Schubarth"
}

Zusammenfassung: Im vorliegenden Beitrag wird der Frage nachgegangen, wie Praxisbezüge im Allgemeinen und Praktika im Besonderen zur Erhöhung der Berufsbefähigung im Studium beitragen können. Ausgehend von Begriffsklärungen werden verschiedene Formen von Praxisbezügen vorgestellt, darauf aufbauend empirische Forschungsbefunde zur Qualität und Wirksamkeit von Praktika - als eine besondere Form von Praxisbezügen - präsentiert und abschließend einige Folgerungen für eine Optimierung von Praxisbezügen bzw. Praktika im (rechtswissenschaftlichen) Studium abgeleitet. Ziel ist es, den Reformbedarf hinsichtlich des Theorie-Praxis-Verhältnisses auch im rechtswissenschaftlichen Studium sowie Möglichkeiten zu seiner Bewältigung aufzuzeigen.

\section{A. Was sind Praxisbezüge?}

Die Begriffe „Praxis“ und „Praxisbezüge“ werden sowohl in der Fachliteratur als auch im Alltagsverständnis nicht einheitlich verwendet. ${ }^{1}$ Bei der Praxis geht es um die Anwendung „erlernte[r] praktische[r] Fertigkeiten ebenso wie um die Verbindung mit und Erfahrungen in der Berufswelt“. ${ }^{2}$ „Praxisbezug “ kann die unmittelbare Anwendung oder die Überprüfung der Gültigkeit einer Theorie bedeuten. Praxisbezüge zeigen sich z.B. in Lehrveranstaltungen durch das Einbringen von Praxisbeispielen, durch Vorträge von Praktikern oder durch Praktika innerhalb und außerhalb der Hochschule. Die Verknüpfung von Theorie und Praxis ist mitentscheidend für den Studienerfolg, insbesondere wenn man darunter die erfolgreiche Einmündung in den Beruf versteht. Praxisbezügen und Praktika kommen dabei zentrale Bedeutung $\mathrm{zu}$.

Praxisbezüge sind - ebenso wie Forschungsbezüge - wesentliche Elemente der Hochschulbildung. Forschung generiert wissenschaftliches Wissen, das in der Praxis angewendet werden soll. Bei akademischen Berufen sind beide Elemente häufig miteinander verzahnt, wenn z.B. Forschung das unmittelbare Praxisfeld darstellt. Auch die Befähigung zum wissenschaftlichen Arbeiten braucht grundsätzlich beide Elemente, Forschungs- und Praxisbezüge, wobei deren Gewichtung unterschiedlich sein kann. Doch herrschen in dieser Hinsicht offenbar größere Defizite, werden doch die Hochschulen, insbesondere die Universitäten, wegen ihres mangelnden Praxisbezuges seit langem kritisiert. $^{3}$

* Der Autor ist Professor für „Erziehungs- und Sozialisationstheorie“ am Profilbereich Bildungswissenschaften der Universität Potsdam.

1 Vgl. dazu ausführlicher Hessler/Oechsle/Scharlau (Hrsg.), Studium und Beruf, Studienstrategien - Praxiskonzepte - Professionsverständnis.

2 Vgl. Bundesministerium für Bildung und Forschung/Multrus, Forschung und Praxis im Studium, S. 10.

3 Vgl. z.B. Schubarth/Speck et al., in: dies. et al. (Hrsg.), S. $47 \mathrm{ff.}$ 
Tabelle 1 gibt einen Überblick über die Formenvielfalt von Praxisbezügen im Rahmen der Hochschule (vgl. Tab. 1). Diese reichen von eher forschungsorientierten Zugängen über lehr- sowie dialogorientierte Zugänge, praxisorientierte Angebote, die eigentlichen Praktika bis hin zu studentischen Initiativen, z.B. in Form von Unternehmensgründungen. Neben der alltäglichen Hochschullehre gibt es auch spezielle Angebote zur Ausbildung von Schlüsselkompetenzen, z.B. zum Wissenstransfer, Projektmanagement oder zur interdisziplinären Zusammenarbeit. Dabei wird häufig übersehen, dass die Hochschule als Institution selbst ein Praxisfeld darstellt. Die Hochschule besitzt dabei einen doppelten Praxisbezug: sowohl intern als Wissenschaftssystem mit der Praxis von Lehre und Studium als auch einen externen Praxisbezug unterschiedlicher Reichweite, z.B. zu Gesellschaft, Arbeitsmarkt, Berufsfeld oder Profession. ${ }^{4}$

\section{Tab. 1: Formen von Praxisbezügen im Studium}

\begin{tabular}{lll} 
Formen & Beispiele & \\
\hline Forschung & Praxis als Gegenstand von Forschung & Praxisforschung \\
\hline Lehre & Praxis als Thema in der Lehre & Fallbasierte Lehre \\
& Texte, Statistiken, Filme über Praxis & Erkundung, Beobachtung \\
& Reflexion von Fallstudien & Interviews mit Praktikern \\
& & Lehre von Praxisvertretern \\
\hline Dialog & Dialogformen & Theorie-Praxis-Workshops \\
& Dialog von Wissenschaftlern und & Projektstudium \\
& Praktikern & \\
\hline Praxis & Praxisformen, Praxisseminare, & Hospitations-, Tages-, Blockpraktika \\
& Rollenspiele & \\
& Praxiserprobungen innerhalb der & Praktika, Praxissemester \\
& Hochschule, & und andere Formen \\
\hline Sonstiges & Studentische Initiativen & \\
\hline
\end{tabular}

Wie Tabelle 1 zeigt, bilden Theorie und Praxis im Studium keinen Gegensatz, durchdringen und ergänzen sich vielmehr gegenseitig. Wildt (2012) hat in diesem Zusammenhang ein Stufenmodell einer Didaktik des Praxisbezugs entwickelt. ${ }^{5}$ Das Modell versucht über verschiedene Stufen hinweg die Praxis von Lehre und Studium durch entsprechende Lernsituationen mit der Praxis des Beschäftigungsfeldes und der Profession zu verbinden und gleichzeitig beispielhafte Lehr-Lern-Formate zu erläutern. Ausgangspunkt stellt das praktische Handeln selbst dar, das durch einen institutionellen Rahmen und eine entsprechende Anleitung ein „Praktikum“ konstituiert. Durch anspruchsvolle hochschuldidaktische Gestaltungen, wie z.B. „problem-based

4 Vgl. Wildt, in: Merkt/Mayrberger (Hrsg.), S. 59 ff.

5 Vgl. Wildt, in: Schubarth/Speck et al. (Hrsg.), S. $261 \mathrm{ff}$. 
teaching “ oder „discovery learning“, können die Anforderungen und Tätigkeiten im Rahmen eines Praktikums systematischer mit dem theoretischen Wissen verknüpft werden. ${ }^{6}$ Eine zusätzliche Herausforderung besteht darin, die Kontexte des Handelns in die Lehr-Lern-Arrangements einzubeziehen, d.h. Problemstellungen, Handlungsstrategien und Erfahrungstatbestände in umfänglichere soziale, kulturelle oder gesellschaftliche Kontexte einzubetten. Dafür eignen sich insbesondere Fallstudien, das Projektstudium oder Formen des Forschenden Lernens. ${ }^{7}$

\section{Wie können Praxisbezüge im Hochschulalltag realisiert werden?}

Zunächst bieten in der Lehre sowohl klassische als auch spezielle Lehrveranstaltungsformate viele Möglichkeiten für einen Praxisbezug. Als klassische Formate gelten Vorlesungen, Seminare und Übungen. Die Vorlesung versteht sich meist als Frontalvortrag zu einem spezifischen Fachgebiet. Die vorgetragenen Lerninhalte werden anschließend von den Studierenden selbstständig anhand vorgegebener Lehr-Lernmaterialien vertieft. So wird primär die Fachkompetenz in dieser Veranstaltungsform gefördert. Bei der didaktischen Gestaltung der Vorlesung ist zu entscheiden, ob das Thema von der Theorie oder von den praktischen Beispielen her vermittelt werden soll: deduktiv, vom Abstrakten zum Konkreten, oder umgekehrt, d.h. induktiv, von anschaulichen Beispielen hin zu einer theoretischen Deutung des Themas. Ausgehend von einer studierendenzentrierten Lehre und einer subjektiven „Verstehenslogik“ der Lernenden ist eher ein induktives Vorgehen zu empfehlen. Häufig wird eine Vorlesung mit obligatorischen Übungen und Seminaren verknüpft, in denen inhaltliche Kenntnisse vertieft oder ein komplexes Thema in Form von Referaten oder schriftlichen Arbeiten wissenschaftlich aufbereitet werden können. In dieser Form werden vor allem die Fach-, Methoden- und Sozialkompetenz gefördert. Die Lernmotivation der Studierenden wird nicht nur dadurch erhöht, dass theoretische Erkenntnisse an praktischen Beispielen verdeutlicht werden, sondern auch dadurch, dass an den Praxiserfahrungen der Studierenden angeknüpft und ein Berufsbezug hergestellt wird. ${ }^{8}$ Auch E-Learning-Elemente wie Online-Tutorien und Hilfefunktionen, interaktive Aufgabenstellungen, Tele-Teaching sowie Lerntagebücher können den Anwendungsbezug in klassischen Präsenzveranstaltungen unterstützen.

Von den speziellen hochschuldidaktischen Ansätzen bzw. Formaten, die Praxisbezüge fördern, sei z.B. auf das Situierte Lernen sowie das Problemorientierte Lernen verwiesen. Der Praxisbezug beim Situierten Lernen ist vor allem dadurch gegeben, dass die Problemstellung vom Lernenden als realistisch, authentisch und lebensnah wahrgenommen wird. Nicht die Reproduktion von Wissen steht im Vordergrund,

6 Vgl. z.B. Forster/Winteler, in: Engelhardt-Nowitzki (Hrsg.),S. 192 ff.; und Hawelka, in: dies./Hammerl et al. (Hrsg.), S. $45 \mathrm{ff}$.

7 Vgl. z.B. Kruse, in: sozial Extra, Durchblick: Projektstudium, Heft 2 (2009), S. 42 ff.; Mertens, Schlüsselkompetenzförderung; Schneider/Wildt, in: Roters/Schneider et al. (Hrsg.), S. 8 ff. und Speck/Wulf et al., in: Schubarth/ders. et al. (Hrsg.), S. $287 \mathrm{ff}$.

8 Vgl. z.B. Marks/Thömen, in: Berendt/Voss et al. (Hrsg.), C 1.1; Kopf/Leipold et al., Kompetenzen in Lehrveranstaltungen und Prüfungen. 
sondern die Anwendung des Gelernten außerhalb der Lernsituation. Deshalb sollen der Kontext und die Situation des Lernenden besondere Berücksichtigung finden. Durch Artikulation und Reflexion im sozialen Austausch soll das neue Wissen in einen Anwendungskontext eingebunden werden, z.B. durch Fallbeispiele. Auch das Problemorientierte oder Problembasierte Lernen, ein selbstgesteuerter Lernprozess mit konkreten Anwendungsbezügen in Kleingruppen, trägt dazu bei, theoretische und praktische Aspekte zu verknüpfen, die Lernmotivation sowie die kommunikativen und sozialen Kompetenzen der Studierenden zu fördern. So steht beim Problemorientierten Lernen stets ein reales Problem oder ein Fall aus der Berufspraxis im Mittelpunkt. Die Studierenden lernen in vorgegebenen Schritten, Informationen zu erschließen, Lösungswege und Ergebnisse zu erarbeiten und zu vergleichen, während ein Tutor den ganzen Gruppenprozess moderiert ${ }^{9}$

\section{B. Praktika als (potenziell) intensivste Form der Theorie-Praxis-Verknüpfung}

Praktika stellen eine besondere Form der Theorie-Praxis-Verknüpfung im Studium dar. Als „vorübergehende Versetzung in die Berufswirklichkeit“"10 werden Praktika eingesetzt, um Kompetenzen zu vermitteln, die in Lehrveranstaltungen nicht oder in nicht ausreichendem Maße erworben werden. Es handelt sich hierbei um „eine Studienform, die im zeitlichen und konzeptionellen Bezug zum Studium steht, dessen Lernzielen und dessen Qualitätsansprüchen folgt “. ${ }^{11}$ Praktika werden von Studierenden sehr geschätzt und meist als zentrale „Strategie der beruflichen Qualifizierung " genutzt. ${ }^{12} \mathrm{Im}$ Folgenden wird insbesondere auf Praktika eingegangen, deren organisatorischer Referenzrahmen außerhalb der Hochschule liegt.

Die Herausforderung eines solchen Praktikums besteht darin, die unterschiedlichen Handlungslogiken der Lernorte Hochschule und Praktikumseinrichtung so zu verknüpfen, dass das Praktikum zum Erreichen der Studienziele beiträgt. Dazu müsse das Praktikum inhaltlich und methodisch in das Studiencurriculum eingebunden sein. Zeitliche, organisatorische und konzeptionelle Bezugspunkte müssen im $\mathrm{Zu}$ sammenhang mit der Zielsetzung des Praktikums berücksichtigt werden. Praktika können unterschiedliche Funktionen erfüllen, z.B. Selbstvergewisserung über den Berufswunsch, Erkundung und Orientierung im Berufsfeld, Anwendung des Gelernten und Erprobung der eigenen Kompetenzen, spezifischer Kompetenzzuwachs oder Kontaktaufnahme für den künftigen Berufsstart sowie Aufbau eines Netzwerkes. Zudem können im Praktikum biographische Bezüge hergestellt werden, die eine Auseinandersetzung mit der eigenen Person sowie der zukünftigen beruflichen Rolle anregen. Insofern kommt dem Praktikum eine besondere Bedeutung als eigenständige Statuspassage innerhalb des Hochschulsozialisationsprozesses zu.

9 Vgl. Marks/Thömen, in: Berendt/Voss et al. (Hrsg.), C 1.1; Wild/Wild, in: Berendt/Voss et al. (Hrsg.), A 2.1.; Oelkers/Kraus, in: ZDRW 2014, S. $142 \mathrm{ff}$.

10 Vgl. Weil/Tremp, in: Berendt/Voss et al. (Hrsg.), S. 1 (2).

11 Vgl. Arbeitsstelle für Hochschuldidaktik der Universität Zürich, S. 7.

12 Vgl. Bloch, in: Beiträge zur Hochschulforschung 29, Heft 4 (2007), S. 82 (89). 
Ein Hauptziel des Praktikums ${ }^{13}$ ist es, das wissenschaftliche Tun an Lernorten außerhalb der Hochschule einzuüben und anschlussfähig zu machen. Dabei spielen sowohl die Reflexion und Systematisierung von Erfahrungen als auch die kritische Betrachtung der Praxis eine Rolle. Von einem hochschuldidaktischen Lehr-Lern-Arrangement kann man in diesem Sinne deshalb erst sprechen, wenn das Praktikum Anleitung und Reflexion erfährt. Zudem kann die Betreuung durch eine qualifizierte Fachperson während des Praktikums wichtig sein. Entsprechend der unterschiedlichen Zielsetzungen lassen sich auch verschiedene Organisationsformen von Praktika finden, wie Kurz-, Block- oder Tagespraktika bis hin zu Praxissemestern. Hinsichtlich der zeitlichen Dimension beschränken sich die Gestaltungsspielräume der Hochschule weitestgehend auf Praktika während des Studiums. Vor dem Studium angesiedelte Praktika können zur Prüfung der Eignung oder Motivation der Studierenden beitragen, während obligatorische Praxisphasen nach dem Studium, wie sie z.B. in der Medizin, Rechtswissenschaft oder der Lehrerbildung verbreitet sind, auf eine Ausübung der beruflichen Tätigkeit vorbereiten sollen. Des Weiteren werden das angestrebte Anspruchsniveau sowie die Lernziele durch die zeitliche Platzierung im Studium und die Dauer des Praktikums bestimmt.

Als ein Qualitätskriterium für Praktika könne deren didaktisch-curriculare Konzeptualisierung gelten. ${ }^{14}$ Um den „Dualismus von Theorie und Praxis“ zu überwinden, solle der Anwendungstransfer bereits an der Hochschule vorbereitet werden, z.B. durch Fallstudien, Simulationen und Planspiele. Im Praktikum übernehmen Betreuer die Rolle von „Transferpaten“. Eine gute Abstimmung zwischen Hochschule und Praktikumseinrichtung, z.B. durch Kooperationsvereinbarungen, sei dabei wichtig. Diskrepanzerlebnisse zwischen Anforderungen und verfügbaren Kompetenzen sollen als Lernanlässe produktiv genutzt werden. Transferprobleme ergeben sich zum einem bei der Übertragung des vorhandenen Wissens auf spezifische Anwendungssituationen (Anwendungsproblem), zum anderen bei der Verallgemeinerung singulärer Lernerfahrungen (Integrationsproblem). Der Reflexion der Praxiserfahrungen während und nach dem Praktikum komme daher große Bedeutung zu, sollen die gemachten Erfahrungen doch systematisiert und auf wissenschaftliche Konzepte bezogen werden. Nach Ruf biete ein Praktikumsbericht Möglichkeiten, formelle wie informelle Lernerfahrungen zu reflektieren und zu dokumentieren. Diese Praxiserfahrungen sollen in den sich anschließenden Lehrveranstaltungen aufgegriffen und in den weiteren Lernprozess integriert werden. Die Annahme, dass eine systematische Verzahnung von Studien- und Praxisphasen, z.B. durch vorbereitende, begleitende und/oder nachbereitende Veranstaltungen wichtig für einen erfolgreichen Lernprozess sei, führt zu der Forderung, dass Praxisphasen „als ein verbindlicher integraler Studienbaustein in die Studienstruktur eingewoben und nicht als fakultative und damit häufig als unkoordinierte (Zusatz-)Komponente konzipiert werden. ${ }^{15}$ Insbeson-

13 Vgl. ausführlicher Schubarth/Speck et al., in: dies. et al. (Hrsg.), S. $47 \mathrm{ff}$.

14 Vgl. im Folgenden Ruf, in: Das Hochschulwesen 54, Heft 4 (2006), S. 135 ff.

15 Vgl. Ruf, in: Das Hochschulwesen 54, Heft 4 (2006), S. 135 (139). 
dere Fachhochschulen und duale Studiengänge zeigen, wie dies in der Praxis umgesetzt werden kann.

\section{Können Praktika zur Erhöhung der Berufsbefähigung im Studium beitragen?}

Zur Beantwortung der Frage nach dem Beitrag von Praktika zur Berufsbefähigung sollen Ergebnisse des Projekts „Professionalisierung von Praxisphasen in außeruniversitären Lernorten“ (ProPrax) vorgestellt werden. Ziel des vom Bundesministerium für Bildung und Forschung (BMBF) geförderten Projekts (Laufzeit von 2009 bis 2012) war es, einen Beitrag zur Professionalisierung von Praxisphasen zu leisten, indem die Qualität und die Wirksamkeit von Praxiskonzepten in unterschiedlichen Fachdisziplinen untersucht und Optimierungsansätze abgeleitet wurden. Schwerpunktmäßig wurden fünf Fachrichtungen an unterschiedlichen Standorten und Hochschularten einbezogen (Agrarwissenschaft, Erziehungswissenschaft, Informatik und Lehramt). Der multiperspektivische Ansatz der Studie umfasste unterschiedliche methodische Zugänge: Dokumentenanalysen, längsschnittliche Befragungen von Studierenden (Selbsteinschätzung) vor und nach dem Praktikum, retrospektive Befragung der Praktikumsbetreuerinnen und -betreuer (Fremdeinschätzung), Expertengespräche mit Praktikumsverantwortlichen sowie Gruppendiskussionen mit Studierenden.

Im Folgenden sollen einige Hauptergebnisse der Studie kurz zusammengefasst werden, ${ }^{16}$ wobei die angeführten Befunde im Einklang stehen mit ähnlichen Befunden zu Praxisphasen und Praxisbezügen der Hochschulforschung insgesamt. ${ }^{17}$

- Die Verankerung von Praxisphasen im Studium unterscheidet sich je nach Hochschulart: Während an Fachhochschulen eine Einbindung meist stattfindet, ist diese bei universitären Studiengänge eher selten (Ausnahme: Lehramt). Bei der Umstellung auf die BA- und MA-Studiengänge hat zwar eine formale Curricularisierung der Praxisphasen an Universitäten stattgefunden, eine Zunahme inhaltlicher wie organisatorischer Vorgaben (z.B. Praktikumsordnung) ist jedoch nicht erkennbar. Neben einer tendenziellen Verkürzung von Praktika zeigt sich, dass die Kompetenzziele in den Modulbeschreibungen universitärer Praxisphasen meist unklar bleiben. Praxisphasen sind je nach Hochschulart und Fachkultur curricular und organisatorisch unterschiedlich konzipiert. Studienprogramme mit klarem Professionsbezug, wie z.B. Rechtswissenschaft oder Lehramt, zielen auf berufliche Identität und professionelle Handlungskompetenz. Praktika in Studiengängen mit klar beschreibbaren Berufsfeldern, wie agrarwissenschaftliche oder Informatikstudiengänge, fokussieren eher auf die Einübung konkreter berufsrelevanter Tätigkeiten. Praktika in Studienrichtungen mit breit gefächerten Berufsfeldern, z.B.

16 Vgl. ausführlicher Schubarth/Speck et al., in: dies. et al. (Hrsg.), S. $47 \mathrm{ff}$.

17 Vgl. z.B. Bargel, in: Schubarth/Speck et al. (Hrsg.), S. 37 ff.; Briedis/Heine et al., Mit dem Bachelor in den Beruf; Heine, in Schubarth/Speck et al. (Hrsg.), S. 45 ff.; Bundesministerium für Bildung und Forschung/Multrus, Forschung und Praxis im Studium; Winter, in: Schubarth/Speck et al., S. 7 ff.; Wolter/ Banscherus, in: Schubert/Speck et al. (Hrsg.), S. $21 \mathrm{ff}$. 
Geisteswissenschaften, sind vor allem auf die berufliche Orientierung und auf die Gewinnung grundlegender Einblicke in die Berufswelt ausgerichtet.

- Auch hinsichtlich des Theorie-Praxis-Verhältnisses unterscheiden sich die Hochschularten beträchtlich: Während Studierende an Fachhochschulen den Praxisbezug in ihrem Studium überwiegend als realisiert ansehen, empfinden drei Viertel der Studierenden diesen an Universitäten generell als zu gering. Die betrachteten universitären Fachkulturen unterscheiden sich dabei kaum; lediglich die Studierenden der Informatik schätzen das Theorie-Praxis-Verhältnis ihres Studiums weniger unausgewogen ein. Die Diskrepanz zwischen der Relevanz, die Studierende den Praxisbezügen im Studium zuschreiben $190 \%$ aller Studierenden halten Praxisphasen für wichtig) einerseits und der mangelnden „Praxistauglichkeit“ des universitären Studiums andererseits wird dadurch evident.

- Praxisphasen haben aus Studierendensicht nachweislich eine berufsorientierende Wirkung. Bei professionsbezogenen Studiengängen wie dem Lehramt ist diese Wirkung am größten. Neben der Berufsorientierung lässt sich ein signifikanter Zuwachs auch bei der berufsbefähigenden Wirkung der Studierenden nachweisen, insbesondere im Bereich der fachlichen Kompetenzen. Als förderliche Faktoren für eine berufsorientierende wie berufsbefähigende Wirkung der Praxisphasen erweisen sich die Betreuungsqualität, der Praxisbezug der (begleitenden) Seminare sowie die individuellen Voraussetzungen der Studierenden. Je klarer die eigenen beruflichen Zukunftsvorstellungen sind, desto größer ist auch die nachgewiesene Wirkung. Ähnliches gilt für die selbst wahrgenommene Kompetenzentwicklung, wobei hier vor allem die Selbstwirksamkeitserwartung bedeutsam ist. Hinsichtlich der Betreuung gibt nur etwa die Hälfte der Studierenden an, regelmäßig einen festen Ansprechpartner an der Hochschule zu haben. Studierende an Fachhochschulen können dabei häufiger einen festen Ansprechpartner vorweisen. Dagegen wird die Betreuung in den Praktikumseinrichtungen über alle Hochschularten und Fachkulturen hinweg als gut eingeschätzt. Dennoch geben sich knapp 15\% der Studierenden nicht mit der Praktikumsanleitung vor Ort zufrieden. Aus Sicht der Mentorinnen und Mentoren vor Ort ist es um die Zusammenarbeit zwischen Praktikumseinrichtungen und Hochschulen nicht allzu gut bestellt. Sie fühlen sich - unabhängig von der Hochschulart - bei der Betreuung ihrer Praktikantinnen und Praktikanten von der jeweiligen Hochschule oft im Stich gelassen.

\section{Empfehlungen für die Optimierung von Praxisbezügen bzw. Praktika}

Aufbauend auf den Befunden des ProPrax-Projekts sollen im Folgenden fünf Empfehlungen vorgestellt werden. Da Praxisphasen nicht isoliert gesehen werden dürfen, zielen einige Anregungen darauf ab, den Praxisbezug im gesamten Studiengang entsprechend zu stärken. ${ }^{18}$

18 Vgl. im Folgenden Schubarth/Speck et al., in: dies. et al. (Hrsg.), S. $47 \mathrm{ff}$. und Schubarth/Kopp et al., in: Das Hochschulwesen, 61/4 (2014), S. $110 \mathrm{ff.}$ 


\section{Empfehlung 1: Praxisbezüge und Praxisphasen curricular integrieren}

Hochschulen sind gefordert, die „Praxistauglichkeit“ des Studiums ${ }^{19}$ zu verbessern. Praxisphasen sollten curricular integriert werden, d.h., in ein Theorie-Praxis-Konzept eingebettet sein, das modulübergreifend Praxisbezüge ermöglicht.

Aus den Ergebnissen des ProPrax-Projekts geht hervor, dass Studierende sich mehr Praxisbezug in ihrem Studium wünschen. Ein praxisbezogenes Studium besteht aus mehr als nur ein oder zwei Pflichtpraktika und erfordert, Handlung und Reflexion zu verknüpfen sowie Erfahrungslernen in Bezug zum Theoriewissen zu setzen. Dies bedeutet, Praxisphasen konzeptionell einzubinden. Um als Studienleistung legitimiert zu werden, müssen daher im Rahmen eines Pflichtpraktikums Kompetenzen erworben werden, die das Erreichen der Studienziele im Allgemeinen unterstützen. Hierbei handelt es sich um Kompetenzen, die nicht oder nur ungenügend in den Lehrveranstaltungen vermittelbar (z.B. Schlüsselkompetenzen) und deshalb entsprechend auszuweisen sind. Wie die Ergebnisse der Dokumentenanalyse zeigen, ist dies in den untersuchten Studiengängen noch kaum erfolgt.

\section{Empfehlung 2: Die berufsorientierende Wirkung von Praxisphasen gezielt unter- stützen}

Keine Praxisphase sollte ohne eine qualifizierte Begleitung durch die Hochschule absolviert werden. Aus organisatorischer und didaktischer Sicht empfiehlt es sich, zur Begleitung und Beratung von Praxisphasen Lehrveranstaltungen einzurichten. Diese sollten in ihrer je spezifischen Zielstellung und Funktion in eine konzeptionelle Passung zum Praktikum gebracht sein.

Praktika unterstützen vor allem die Berufsorientierung und Berufsbefähigung der Studierenden und zwar gleichermaßen an Universitäten wie Fachhochschulen. Einfluss auf die berufliche Orientierung hat zuvorderst die Betreuung während des Praktikums in der Praktikumseinrichtung und an den Hochschulen. Die Betreuung und Begleitung in den Hochschulen wird, wenn diese überhaupt erfolgt, kritisch eingeschätzt. Dies gilt insbesondere mit Blick darauf, inwieweit begleitende Veranstaltungen stattfinden bzw. inwieweit diese einen ausreichenden Praxisbezug herstellen.

\section{Empfehlung 3: Qualität der Praktikumsbetreunng verbessern}

Die Qualitätssicherung der Ausbildung an den Praktikumseinrichtungen trägt maßgeblich dazu bei, die Wirkung von Praxisphasen zu erhöhen. Deshalb sollten Standards für die Anerkennung von Praktikumseinrichtungen vereinbart, die fachliche Anleitung und Betreuung am Praktikumsort gesichert und gegebenenfalls Maßnahmen zur Qualifizierung der Mentorinnen und Mentoren angeboten werden.

Für die Erhöhung von Berufsbefähigung sind theoretische und praktische Ausbildungsanteile aufeinander abzustimmen, um einen Theorie-Praxis-Transfer zu er- 
möglichen. Auf der einen Seite bieten sich in den Praktikumseinrichtungen viele Einsatzgebiete, auf der anderen Seite bedeutet dies nicht automatisch tatsächliche Beobachtungs- und Erprobungsmöglichkeiten unter fachlicher Anleitung für die Studierenden. Ergebnisse der Dokumentenanalyse legen nahe, dass hier z.B. Verträge und gegenseitige Zielvereinbarungen zu institutioneller Verbindlichkeit und Transparenz beitragen, um entsprechend qualifizierte Einsatzmöglichkeiten für die Studierenden zu sichern. Dazu gehört auch, Standards für Praktikumseinrichtungen zu entwickeln und die Mentorinnen und Mentoren vor Ort zu qualifizieren.

\section{Empfehlung 4: Kooperation mit Praktikumseinrichtungen ausbauen}

Praxisphasen sind in Zusammenarbeit mit allen Beteiligten (Hochschulen - Studierende - Praktikumseinrichtungen) zu gestalten. Dazu sollten Erwartungen und unterschiedliche Perspektiven kommuniziert, Rahmenbedingungen und Ressourcen der Hochschulen und Unternehmen geprüft, Studieninhalte und Praktikumsziele aufeinander abgestimmt werden.

Praxisphasen sollten nicht im „Alleingang“ gestaltet werden, sondern im Dialog und durch die Zusammenarbeit aller Beteiligten. Bisher aber gibt es noch kaum Untersuchungen darüber, was Hochschulverantwortliche in Praktika und was Praktikumsanbieterinnen und -anbieter gegenüber den Hochschulen hinsichtlich der Ausgestaltung von Praktika erwarten. Ohne eine gemeinsame Klärung der Erwartungen und unterschiedlichen Perspektiven lassen sich Praxisphasen jedoch nur schwer optimieren. Wie die Ergebnisse von ProPrax zeigen, klagen die Unternehmen sowie die Mentorinnen und Mentoren über eine unzureichende Kooperation mit den Hochschulen, das gilt für Universitäten und Fachhochschulen gleichermaßen.

\section{Empfehlung 5: Qualität von Praxisbezügen und Praxisphasen sichern}

Praxisphasen und Praxisbezüge sollten eine Aufwertung im Studium durch die Hochschule erfahren und entsprechend Eingang in die Studiengangentwicklung finden. Dafür ist sowohl auf der Ebene der Hochschulleitung als auch unter den Hochschullehrenden und Dozierenden Akzeptanzmanagement zu betreiben. Als wichtige Rahmenbedingung sind eine angemessene Deputatsanrechnung für die Betreuung von Praxisphasen sowie geeignete Anreizsysteme (wie z.B. Zielvereinbarungen) erforderlich.

Der Weg von Empfehlungen in die Hochschulpraxis führt in der Regel über die entsprechenden Studienprogramme bzw. Studienordnungen. Die systematische Integration von Praxisbezügen und -phasen in einen Studiengang erfolgt allerdings sehr unterschiedlich, wie die Analysen gezeigt haben. Um die Frage zu beantworten, wie Empfehlungen zur Professionalisierung von Praxisphasen Eingang in die Hochschule finden, empfiehlt es sich, auch Gründen nachzugehen, warum an Universitäten, insbesondere in berufsfeldunspezifischen Studiengängen (z.B. auch den Rechtswissen- 
schaften), Widerstände gegen Praxisbezüge und integrierte Praxisphasen existieren. ${ }^{20}$ Beispielsweise bedarf es - nicht zuletzt angesichts wachsender Studierendenzahlen - eines hohen (zusätzlichen) Personal- und Koordinierungsaufwands, um ein Praktikum zu betreuen. Der zuständige Fachbereich und jede seiner Professuren definieren sich an der Universität aber zuvorderst über die Forschung und weniger über die Lehre. Ein im Vergleich zu traditionellen Lehrformen (Vorlesung, Seminar) geringerer curricularer Anrechnungsfaktor für die Praktikumsbetreuung schafft zudem „Nachteile“ in der Deputatsanrechnung bei einem vergleichsweise hohen persönlichen Zeitaufwand. Den akademischen Mittelbau trifft dieses Ungleichgewicht angesichts meist befristeter Stellen besonders. Die Diskussion um Praxisbezüge und Praxisphasen scheint daher eng mit dem Leitbild und dem Selbstverständnis der jeweiligen Hochschule verwoben zu sein.

\section{E. Folgerungen für die Ausbildung in den Rechtswissenschaften}

Ein Blick in die Geschichte macht deutlich, dass das Theorie-Praxis-Verhältnis innerhalb der Ausbildung in den Rechtswissenschaften schon seit längerem kontrovers diskutiert wird. Bereits in den 1970er Jahren wurde im Zuge von Reformdiskussionen die große Distanz zwischen Wissenschaft und Praxis kritisiert. Im Fokus der Kritik standen die unzureichende wissenschaftliche Aufarbeitung gesellschaftlicher Konflikte und deren rechtliche Regelung. Vor diesem Hintergrund wurde eine engere Kopplung zwischen wissenschaftlich angeleiteter Ausbildung und Praxis eingefordert. Anschließende Reformbestrebungen in den Ländern und Hochschulen zielten auf eine neue, einphasige Juristenausbildung ab, die ein verändertes Curriculum, eine verstärkte Einbindung der Sozialwissenschaften und neue Lehr- und Lernformen beinhaltete. Die im Anschluss daran entstandenen Modelle in den Ländern verbanden Theorie- und Praxiselemente deutlich enger miteinander (Einphasigkeit) und integrierten die Sozialwissenschaften. Ungeachtet zahlreicher positiver Evaluationsbefunde wurden die Reformprojekte bereits nach wenigen Jahren beendet. Ausschlaggebend hierfür waren nach Zeitzeugenberichten (Haag, Richter) kritische Einwände auf Seiten der Politik gegen eine zu enge Theorie-Praxis-Verknüpfung (vgl. auch die polytechnische Ausbildung in der DDR) und Befürchtungen vor einer Politisierung der Justiz durch die Sozialwissenschaften.

Ungeachtet dessen schneiden die Rechtswissenschaften beim bundesweiten Fächervergleich des Praxisbezugs relativ gut ab. Wie die Studien zeigen, verfügen Studierende in den Rechtswissenschaften auf der einen Seite zwar über eher kurze Praktika und haben eher geringe Erwartungen an Praktika und den Erwerb von praktischen Fähigkeiten im Studium. Auf der anderen Seite bewerten sie - neben den Medizinern - den vorhandenen Praxisbezug im Vergleich aller Fächer am besten. Weniger gut wird jedoch die Vermittlung und Betreuung von Praktika bewertet. ${ }^{21}$

20 Vgl. Schubarth/Speck et al., in: Zeitschrift für Hochschulentwicklung 3/2011, S. 74 ff.

21 Vgl. z.B. AG Hochschulforschung der Universität Konstanz und Bundesministerium für Bildung und Forschung/Multrus, Forschung und Praxis im Studium. 
In jüngster Zeit gibt es allerdings vermehrte Anstrengungen, die Praxisbezüge in den Rechtswissenschaften zu verstärken. Dazu zwei Good-Practice-Beispiele. ${ }^{22}$ An der Universität Köln wird beispielsweise das Projekt „Recht Aktiv“ angeboten. Dieses Lehrangebot verfolgt das Ziel, ab dem ersten Semester Kernkompetenzen, aber auch die Soft Skills und die Motivation der Studierenden zu fördern. Einen zentralen Schwerpunkt stellen simulierte Gerichtsverhandlungen dar. Ein anderes Beispiel ist das Projekt Compliance E-lliance der Universität Leipzig. Compliance E-lliance steht für a) Maßnahmen von Unternehmen zur Einhaltung und Befolgung des geltenden Rechts („Compliance“), b) eine Kommunikation auf elektronischem Wege („E“) sowie c) die Partnerschaft zwischen zwei rechtswissenschaftlichen Fakultäten verschiedener Länder („E-lliance“). In diesem englischsprachigen Lehr- und Prüfungsformat, einem Kooperationsprojekt zwischen der Universität Leipzig und der Miami University Law School, werden praxisrelevante Seminarthemen des Arbeitsfeldes Compliance behandelt und diskutiert. Ziel ist es, die Schlüsselqualifikationen der Studierenden in einem authentischen Arbeitsumfeld und anhand praxisnaher Beratungsszenarien zu verbessern, die Fremdsprachenkompetenz durch Teamarbeit zu steigern, den berufsbezogenen Umgang mit neuen Medien zu erproben, fächerübergreifende und interdisziplinäre Problemlösungsstrategien zu entwickeln sowie neue Fachkenntnisse durch Wissenstransfer zu erschließen.

Wie diese und andere Beispiele zeigen, gibt es zahlreiche Möglichkeiten, Praxisbezüge in das Studium der Rechtswissenschaften zu integrieren. Letztlich kommt es weniger auf den Innovationsgehalt der Praxisbezüge an, als darauf, entsprechende Verknüpfungen sinnvoll herzustellen, d.h. diese gezielt vorzubereiten, zu begleiten und auszuwerten. Von den vielfältigen Bemühungen, Praxisbezüge in den Rechtswissenschaften zu stärken, zeugt nicht zuletzt auch die fachinterne Diskussion um Schlüsselqualifikationen in der rechtswissenschaftlichen Ausbildung (z.B. Kommunikation, Gesprächsführung, Mediation, Rhetorik). Diese guten Ansätze gilt es im Kontext hochschuldidaktischer Professionalisierung weiter auszubauen. ${ }^{23}$

\section{Literatur}

AG Hochschulforschung, Studierendensurvey. Universität Konstanz 2012, News 39.3/03.12

AfH $=$ Arbeitsstelle für Hochschuldidaktik der Universität Zürich, Praktikum, Orientierungshilfen für Programm- und Modulverantwortliche, Dossier, Zürich 2010.

Bargel, Tino, Bedeutung von Praxisbezügen im Studium, in: Schubarth/Speck/Seidel/Gottmann/Kamm/ Krohn (Hrsg.), Studium nach Bologna: Praxisbezüge stärken?! Praktika als Brücke zwischen Hochschule und Arbeitsmarkt, Befunde und Perspektiven, Wiesbaden 2012, S. 37-46.

Bloch, Roland, „Natürlich möchte man es auch gern im Lebenslauf stehen haben...“ - Bedeutungen des Praktikums für Studierende, in: Beiträge zur Hochschulforschung 29, Heft 4 (2007), S. 82-107.

Briedis, Kolja/Heine, Christoph/Konegen-Grenier, Christiane/Schröder, Ann-Kathrin, Mit dem Bachelor in den Beruf, Arbeitsmarktbefähigung und -akzeptanz von Bachelorstudierenden und -absolventen 2011, http://www.stifterverband.org/publikationen_und_podcasts/positionen_dokumentationen/ mit_dem_bachelor_in_den_beruf/mit_dem_bachelor_in_den_beruf.pdf. (18.4.2014).

22 Vgl. ausführlicher Schubarth/Speck, Employability und Praxisbezüge im wissenschaftlichen Studium.

23 Vgl. Reis, in: ZDRW 2013, S. $21 \mathrm{ff}$. 
Bundesministerium für Bildung und Forschung/Multrus, Frank, Forschung und Praxis im Studium, Befunde aus Studierendensurvey und Studienqualitätsmonitor, Bonn/Berlin 2012.

Forster, Peter/Winteler, Adi, Vom Lehren zum Lernen: Ein neues Paradigma für die Hochschullehre, in: Engelhardt-Nowitzki (Hrsg.), Ausbildung in der Logistik, Wiesbaden 2006, S. 192-210.

Hawelka, Birgit, Problemorientiertes Lehren und Lernen, in: dies./Hammerl/Gruber (Hrsg.), Förderung von Kompetenzen in der Hochschullehre, Theoretische Konzepte und ihre Implementation in der Praxis, Kröning 2007, S. 45-58.

Heine, Christoph, Studienqualität nach Bologna aus Studierendensicht, Wahrnehmung und Bewertung von Studienbedingungen und Praxisbezug, in: Schubarth/Speck/Seidel (Hrsg.), Nach Bologna: Praktika im Studium - Pflicht oder Kür? Empirische Analysen und Empfehlungen für die Hochschulpraxis, Potsdam 2011, S. 45-78.

Hessler, Gudrun//Oechsle, Mechtild/Scharlau, Ingrid (Hrsg.), Studium und Beruf, Studienstrategien - Praxiskonzepte - Professionsverständnis, Bielefeld 2013.

Kopf, Martina/Leipold, Jana/Seidl, Tobias, Kompetenzen in Lehrveranstaltungen und Prüfungen, Handreichung für Lehrende, Mainzer Beiträge zur Hochschulentwicklung Bd. 16, Mainz 2010.

Kruse, Elke, Projektstudium und Praxisbezüge im Bologna-Prozess: Reform der Reform?, in: sozial Extra, Durchblick: Projektstudium, Heft 2 (2009), S. 42-47.

Marks, Frank/Thömen, Doris, Die Moderation des Problemorientierten Lernens (POL), Die Rekonstruktion der Wirklichkeit, in: Berendt/Voss/Wildt (Hrsg.), Neues Handbuch der Hochschullehre, Berlin 2002, C 1.1.

Mertens, Claudia, Schlüsselkompetenzförderung, Bielefeld 2008.

Oelkers, Janine/Kraus, Philip Alexander, Problembasiertes Lernen (PBL) in der rechtswissenschaftlichen Lehre, in: ZDRW 2014, S. 142-149.

Reis, Oliver, Perspektiven rechtswissenschaftlichen Studiums, in: ZDRW 2013, S. 21-43.

Ruf, Michael, Praxisphasen als Beitrag zur Employability, Didaktische Funktionsbestimmung betrieblicher Praxisphasen im Rahmen wirtschaftswissenschaftlicher Bachelor- Studiengänge, in: Das Hochschulwesen 54, Heft 4 (2006), S. 135-139.

Schneider, Ralf/Wildt, Johannes, Forschendes Lernen in Praxisstudien - Wechsel eines Leitmotivs, in: Roters/Schneider/Koch-Priewe/Thiele/Wildt (Hrsg.), Forschendes Lernen im Lehramtsstudium, Hochschuldidaktik - Professionalisierung - Kompetenzentwicklung, Bad Heilbrunn 2009, S. 8-36.

Schubarth, Wilfried/Kopp, Andrea/Seidel, Andreas/Ulbricht, Juliane, Praxisphasen als Beitrag zur Erhöhung der Berufsbefähigung im Studium, Ergebnisse und Empfehlungen des Projekts „Professionalisierung von Praxisphasen in außeruniversitären Lernorten " (ProPrax), in: Das Hochschulwesen, 61/4 (2014), S. 110-113.

Schubarth, Wilfried/Speck, Karsten, Employability und Praxisbezüge im wissenschaftlichen Studium“, Fachgutachten für die HRK, http:/www.hrk-nexus.de/fileadmin/redaktion/hrk-nexus/07-Downloads/ 07-02-Publikationen/Fachgutachten_Employability.pdf (18.4.2014).

Schubarth, Wilfried/Speck, Karsten/Seidel, Andreas/Gottmann, Corinna/Kamm, Caroline/Krohn, Maud (Hrsg.), Studium nach Bologna: Praxisbezüge stärken?! Praktika als Brücke zwischen Hochschule und Arbeitsmarkt, Befunde und Perspektiven, Wiesbaden 2012.

Schubarth, Wilfried/Speck, Karsten/Seidel, Andreas/Gottmann, Corinne/Kamm, Caroline/Krohn, Maud, Praxisbezüge im Studium - Ergebnisse des ProPrax-Projektes zu Konzepten und Effekten von Praxisphasen unterschiedlicher Fachkulturen, in: dies. (Hrsg.), Studium nach Bologna: Praxisbezüge stärken?! Praktika als Brücke zwischen Hochschule und Arbeitsmarkt, Befunde und Perspektiven, Wiesbaden 2012, S. 47-100.

Schubarth, Wilfried/Speck, Karsten/Seidel, Andreas/Gottmann, Corinna/Kamm, Caroline/Kopp, Andreas/ Krohn, Maud, Nach Bologna: Warum das Neue (manchmal) nicht in die Hochschule kommt, Das Beispiel Praxisphasen im Studium, in: Zeitschrift für Hochschulentwicklung 3/2011, S. 74-88, http:// www.zfhe.at/index.php/zfhe/article/view/353/438 (18.4.2014).

Speck, Karsten/Wulf, Carmen/Viertel, Michael/Arnold, Doris/Ivanova-Chessex, Olga, Praxisbezüge im Studium durch „Forschendes Lernen“, in: Schubarth/Speck/Seidel/Gottmann/Kamm/Krohn (Hrsg.), Stu- 
dium nach Bologna: Praxisbezüge stärken?! Praktika als Brücke zwischen Hochschule und Arbeitsmarkt, Befunde und Perspektiven, Wiesbaden 2012, S. 287-298.

Weil, Markus/Tremp, Peter, Praktika im Studium als Berufswirklichkeit auf Zeit, Zur Planung und Gestaltung obligatorischer Praktika im Studium, in: Berendt/Voss/Wildt (Hrsg.), Neues Handbuch Hochschullehre, Berlin 2010, S. 1-16.

Wildt, Johannes, Praxisbezug revisited - Zur hochschuldidaktischen Rekonstruktion von Theorie-PraxisVerhältnissen in Studium und Lehre, in: Merkt/Mayrberger (Hrsg.), Die Qualität akademischer Lehre, Zur Interdependenz von Hochschuldidaktik und Hochschulentwicklung, Innsbruck 2007, S. 59-72.

Wildt, Johannes, Praxisbezug der Hochschulbildung - Herausforderung für Hochschulentwicklung und Hochschuldidaktik, in: Schubarth/Speck/Seidel/Gottmann/Kamm/Krohn (Hrsg.), Studium nach Bologna: Praxisbezüge stärken?! Praktika als Brücke zwischen Hochschule und Arbeitsmarkt, Befunde und Perspektiven, Wiesbaden 2012, S. 261-278.

Wild, Elke/Wild, Klaus-Peter, Jeder lernt auf seine Weise... Individuelle Lernstrategien und Hochschullehre, in: Berendt/Voss/Wildt (Hrsg.), Neues Handbuch Hochschullehre, Lehren und Lernen effizient gestalten, Berlin 2002, A 2.1.

Winter, Martin, Praxis des Studierens und Praxisbezug im Studium. Ausgewählte Befunde der Hochschulforschung zum „neuen“ und „alten“ Studieren, in: Schubarth/Speck/ Seidel (Hrsg.), Nach Bologna: Praktika im Studium - Pflicht oder Kür? Empirische Analysen und Empfehlungen für die Hochschulpraxis, Potsdam 2011, S. 7-43.

Wolter, Andrä/Banscherus, Ulf, Praxisbezug und Beschäftigungsfähigkeit im Bologna-Prozess - „A never ending story“?, in: Schubarth/Speck/Seidel/Gottmann/Kamm/Krohn (Hrsg.), Studium nach Bologna: Praxisbezüge stärken?! Praktika als Brücke zwischen Hochschule und Arbeitsmarkt, Befunde und Perspektiven, Wiesbaden 2012, S. 21-36. 